УДК 550.3

\title{
УЧЁТ ВЛИЯНИЯ ВЕРТИКАЛЬНОЙ ПРОНИЦАЕМОСТИ НА АДАПТАЦИЮ МЕСТОРОЖДЕНИЯ С ТЕРРИГЕННЫМ ТИПОМ КОЛЛЕКТОРА
}

\author{
Коровин Михаил Олегович, \\ korovinmo@hw.tpu.ru \\ Национальный исследовательский Томский политехнический университет, \\ Россия, 634050, г. Томск, пр. Ленина, 30.
}

\begin{abstract}
Актуальность исследования состоит в необходимости создания алгоритма расчёта проницаемости в трёхмерном пространстве по геолого-геосизическим данным. На данном этапе существует возможность учёта влияния анизотропии проницаемости в горизонтальной плоскости. Вертикальная проницаемость, как правило, задаётся соотношением проницаемости в горизонтальной плоскости и понижающим или повышающим коэфффициентом.

Фактически в таком случае не учитьвается пространственное изменение вертикальной составляющей проницаемости по разрезу, что обусловлено незначительным количеством исследований вертикальной проницаемости и соответственно отсутствием уравнений для расчёта этого очень важного параметра. Это глобальная проблема недостатка исходной информации при исследованиях фрактического кернового материала, который является основой для изучения анизотропных характеристик горных пород. Проницаемость, в свою очередь, является важнейшей характеристикой пласта и влияет на возможности добычи уелеводородов из пластов коллекторов.

В цели данной работы входило создание зависимости вертикальной проницаемости от пористости, распределение проницаемости в геологической и гидродинамической модели, проверка качества адаптации данных добычи и закачки.

Объектом исследования является месторождение с терригенным типом коллектора. На данном месторождении уже были проведены расчёты анизотропии горизонтальной проницаемости, а в рамках данного исследования проведены расчёты вертикальной проницаемости и учтено её влияние на адаптацию месторождения. Особенностью данного месторождения является изменчивость направления улучшенных зон проницаемости, а также наличие лабораторных керновых исследований вертикальной проницаемости коллекторов. Такие исследования позволят определить вертикальную проницаемость горных пород и рассчитать распределение по вертикали разреза. Исследуемые пласты представлены верхнеюрскими отложениями с неоднородным распределением фильтрационных и ёмкостных свойств.

Методы: построение зависимости вертикальной проницаемости от пористости по керновым исследованиям, внедрение в геологическую и гидродинамическую модели вертикальной составляющей проницаемости, расчёт адаптации фрактических данных добычи после учёта вертикальной составляющей проницаемости.

В результате исследования была уточнена петрофизическая модель исследуемого месторождения, что отразилось в более точном распределении петрофизических свойств в геологической модели. Таким образом, становится возможным учитьвать реальные особенности геологического строения, так как в трёхмерном пространстве необходимо использовать все три компоненты вектора проницаемости. Одним из главных моментов является создание модели, максимально точно описывающей распределение свойств и отражающей реальные характеристики добычи. Только в этом случае можно проводить наиболее точный прогноз и планировать применение методов увеличения нефтеотдачи. Огромное количество накопленных данных позволяет создавать корреляции между различными петрофизическими параметрами. Необходимо дополнять и нарабатывать объём информации, который в ближайшем будущем может служить базой данных для месторожденийаналогов, у которых отсутствуют данные для создания собственных подобных зависимостей.
\end{abstract}

\section{Ключевые слова:}

Анизотропия проницаемости, масштаб анизотропии, вертикальная анизотропия, гидродинамическое моделирование, петрофизические расчёты.

\section{Введение}

Проблематика текущего состояния разработки заключается в следующем: огромный массив информации уже невозможно держать только в представлении одного или нескольких специалистов, изучающих конкретное месторождение. При количестве скважин, измеряющихся десятками, уже становится сложно ориентироваться во всё увеличивающихся объёмах геолого-геофизической информации. Если брать во внимание месторождения с количеством скважин около 1000 или 10000, то здесь без специализированного программного обеспечения не обойтись. Возможности современного программного обеспечения позволяют не только хранить, но и обрабатывать большие объёмы информации. Дополнительным и очень важным фактором является возможность проводить стандартные расчёты для большого количе- ства скважин, а также реализовывать нестандартные расчёты, требующие выполнения сложных математических операций и последовательности действий.

В настоящее время это применимо в качестве детального учёта фильтрации углеводородов. Проницаемость вычисляется стандартным способом через зависимость проницаемости от пористости по керну. На выходе получается величина проницаемости, которую в дальнейшем распространяют в геологической модели для прогноза добычи нефти. Эта величина проницаемости является усреднённым результирующим вектором проницаемости в каждой точке расчёта. Конечно, отдельной проблемой является не только то, что результирующие вычисления характеризуют лишь усреднённое значение проницаемости, но и однонаправленность вектора в конкретном направлении, в частности, по оси Х. Отдельно хоте- 
лось бы отметить недостаточность данных для проведения соответствующих расчётов. Поэтому такие расчёты и исследования в данном направлении обладают ещё и дополнительной ценностью - полученные уравнения можно использовать на других месторождениях для повышения точности расчётов и распространения фильтрационных свойств. Получается, что выведенные уравнения можно будет использовать в качестве опорных для расчётов свойств на других месторождениях со схожей обстановкой осадконакопления и качеством коллектора, а месторождение можно будет использовать в качестве месторождения-аналога. Набор статистики и формирование базы уравнений очень важная вещь с позиции проведения расчётов на месторождениях с некачественными лабораторными исследованиями керна или вовсе без таковых.

\section{Предпосылки изучения фильтрационной} анизотропии коллекторов

Феномен наличия различных направлений фильтрации флюидов изучается довольно давно [1-16], и исследователи смогли приблизиться к детальному описанию этого феномена - анизотропии проницаемости. Настоящая работа посвящена методике учёта вертикальной компоненты проницаемости. Первым подходом, который планируется предпринять, является создание базовых петрофизических зависимостей с параметром вертикальной проницаемости в качестве оси ординат.

Также немаловажным фактором, как и при проведении исследований горизонтальной проницаемости, является необходимость предварительной ориентировки образцов керна. В этом случае удаётся вычислить не только скалярную компоненту вектора проницаемости, но и определить направление этого вектора.

Дополнительный интерес вызывают работы [2, 3]. Они посвящены изучению околоскважинного пространства и проведению записи изменения физических полей специализированными зондами компании Schlumberger. Эти количественные характеристики интересны ещё и тем, что авторы также затрагивают вопрос определения и оценки анизотропии проницаемости. После проведения интерпретации результатов авторы приходят к интересной мысли, что режим течения в анизотропных средах не является сферическим, как это обычно предполагается при проведении стандартных расчётов. В своей работе авторы не учитывают горизонтальную анизотропию проницаемости, но проводят важные расчёты и делают вывод о том, что в тех пластах, где вертикальная анизотропия наиболее выражена, сила потока флюидов будет находиться под большим влиянием горизонтальной компоненты проницаемости.

Необходимо отметить, что подобные исследования являются серьёзным шагом вперёд при определении свойств пласта при проведении гидродинамических испытаний этого пласта. Это позволяет уже на ранней стадии оценить первичное направление проницаемости и охарактеризовать направление изменения свойств пластов. Но также необходимо помнить, что регистрация этих параметров проводится не в каждой скважине и не всегда имеется возможность сразу оценить явление и направление анизотропии вертикальной проницаемости. Логика применения этих вычислений: после бурения скважины с отбором керна керн передаётся в лабораторию в том числе и для расчёта вертикальной проницаемости. В это же время проводятся замеры и расчёты по методике, предложенной авторами работ $[2,3]$, делаются предварительные выводы о величине и направлении анизотропии вертикальной проницаемости. В дальнейшем, после получения фильтрационно-ёмкостных свойств керна, проводятся лабораторные анализы на керне, а также учёт горизонтальной проницаемости. Следующий шаг - корректировка или подтверждение расчётов по методике авторов [2, 3], которое можно использовать в качестве дополнительного обоснования проведённых расчётов.

В текущих работах по учёту проницаемости и распространения петрофизических свойств коллекторов во внимание принимается горизонтальная проницаемость и коэффициент, позволяющий вычислить вертикальную составляющую проницаемости [4-11]. Во многом это обусловлено отсутствием данных вертикальной проницаемости по керновому материалу. Соответственно, затруднительно сравнить вычисленную вертикальную проницаемость с лабораторными исследованиями керна и распределить расчётные величины проницаемости по вертикали разреза.

Таким образом, проницаемость достаточно достоверно распределена в двух горизонтальных плоскостях, а в вертикальной плоскости расчёты равнозначной детальности отсутствуют. Поэтому первоначальной задачей является выработка методики расчёта и распределения вертикального компонента проницаемости. Начать необходимо с установления петрофизической взаимосвязи ключевых параметров. Речь идёт о пористости и проницаемости. Взаимосвязь между этими параметрами на данном этапе исследования будет иметь ключевое значение.

Классический вариант учёта фильтрационной анизотропии подразумевает введение коэффициента пересчёта горизонтальной проницаемости в вертикальную. Зачастую наблюдается превышение значений горизонтальной составляющей над вертикальной. Однако применяемые коэффициенты пересчёта основаны на эмпирическом представлении о порядке уменьшения вертикальной проницаемости относительно горизонтальной [12-18]. Существуют измерения вертикальной проницаемости по керновым данным при проведении лабораторных исследований. Зачастую эти измерения не проводятся, и вычислить действительные значения вертикальной проницаемости очень сложно [19-22]. Если же такие значения существуют, то становится возможным дополнить стандартную интерпретацию дополнительным типом зависимости петрофизических параметров, а именно вертикальной проницаемости от пористости. Таким образом, становится возможным приблизиться к распределению вертикального вектора проницаемости в разрезе скважины. 


\section{Дополнительные петрофизические расчёты}

Стандартная петрофизическая зависимость ограничивается созданием соотношения типа керн-керн между проницаемостью и пористостью. Базовый подход заключается в том, чтобы вертикальную компоненту проницаемости вычислить в виде умножения горизонтальной компоненты на постоянный коэффициент. На большом количестве нефтяных месторождений вертикальная компонента в численном выражении меньше горизонтальной. Это сложившаяся практика, закономерности которой наблюдаются на большинстве месторождений с терригенным типом коллектора.

Для получения достоверных значений необходимо провести предварительную работу с керновым материалом. Первым шагом необходимо создать зависимость проницаемости от пористости. В классическом подходе это делается для образцов параллельного выпиливания. В данном случае применяется тот же самый подход, но для образцов перпендикулярного выпиливания (рис. 1). В итоге наблюдается очень хорошая зависимость экспоненциального типа с высоким коэффициентом корреляции и низкой дисперсией.

Дополнительно была построена зависимость вертикальной проницаемости от горизонтальной проницаемости по образцам керна (рис. 2). Такая зависимость наглядно показывает различия между компонентами проницаемости. Дополнительно такой подход позволяет выявить уравнение, с помощью которого становится возможным провести расчёт вертикальной проницаемости. Это необходимо на тот случай, когда зависимость вертикальной проницаемости от пористости либо не существует, либо является недостоверной. Проанализировав график, удаётся увидеть, что вертикальная компонента ненамного отличается от горизонтальной компоненты. Получается, что анизотропность пространства коллектора невелика по вертикали. Тем не менее, существующая формула позволяет более точно учесть распределение проницаемости по вертикали разреза.

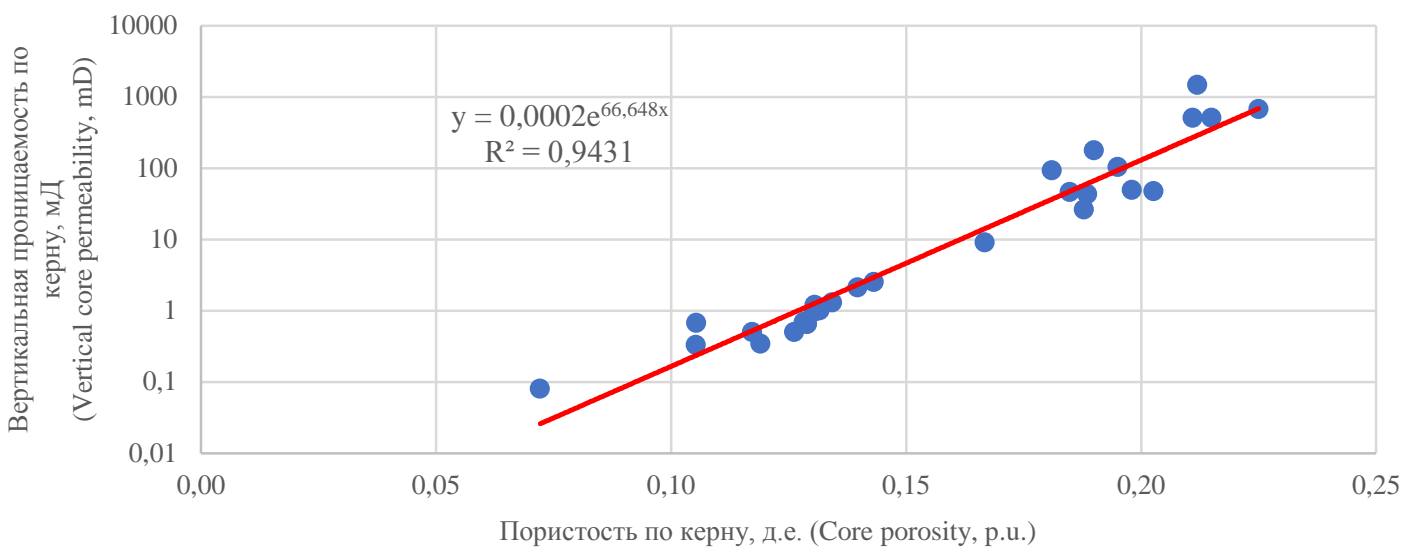

Рис. 1. Зависимость вертикальной проницаемости от пористости по керну

Fig. 1. Vertical permeability porosity relationship

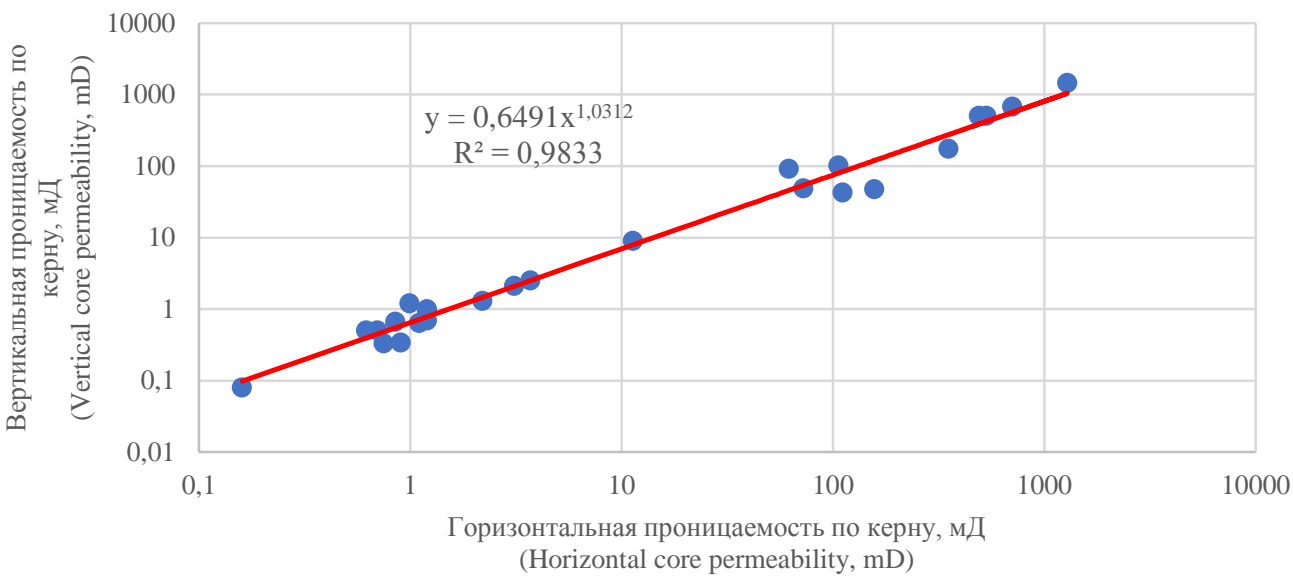

Рис. 2. Зависимость вертикальной проницаемости от горизонтальной проницаемости по керну

Fig. 2. Vertical permeability horizontal permeability relationship

Дополнительно были предприняты попытки создать зависимость вертикальной проницаемости от остаточной водонасыщенности по керну (рис. 3). Следует отметить важные факторы: зависимость су- ществует, коэффициент корреляции высокий. Небольшое количество точек обусловлено недостаточным количеством исследований остаточной водонасыщенности на тех же глубинах, где произведено 
определение вертикальной проницаемости. Данная зависимость создана для понимания принципиального существования подобной взаимосвязи параметров и для получения дополнительного уравнения расчёта вертикальной составляющей проницаемости через параметр, неравнозначный пористости. Вид зависимости не отличается от вида аналогичной зависимости для горизонтальной составляющей. Дополнительно на рис. 3 отражено распределение точек горизонтальной проницаемости и водонасыщенности. Детальный анализ сложно проводить из-за малого количества данных вертикальной проницаемости. Все точки оказываются в области низких значений остаточной водонасыщенности, где преобладает нефтяной флюид. Если рассматривать весь объём исследований горизонтальной проницаемости, то наблюдается изменение коэффициента уравнения а практически в 2,5 раза. Однако, если создать зависимость горизонтальной проницаемости от остаточной водонасыщенности в тех же пределах, в которых есть данные по вертикальной проницаемости (Кво<0.3 д.е.), получается, что зависимости практически совпадают и коэффициенты отличаются друг от друга на небольшие величины. На рис. 3 видно, что линии тренда практически полностью накладываются друг на друга (жёлтая и фиолетовая). Выявляется интересная взаимо- связь параметров в области высокой нефтенасыщенности, но в области повышенной водонасыщенности такие вывод делать пока рано из-за отсутствия дополнительных керновых данных.

Подобный набор графиков (рис. 1-3) полностью характеризует распределение фильтрационных свойств по вертикали разреза. Данные графики и зависимости можно использовать в качестве аналогов для других месторождений. Перед этим необходимо будет разместить точки керна на данной зависимости и проверить, не нарушается ли исходная зависимость. Если точек керна нет, то в первом приближении можно использовать напрямую полученные зависимости при учёте одинаковой обстановки осадконакопления у месторождения, для которого планируется вычислить вертикальную проницаемость. Таким образом, петрофизическая модель дополнена новыми зависимостями. На этот факт делается дополнительный акцент для того, чтобы ярче подчеркнуть необходимость использования всей доступной информации и учёта всех расчётных параметров. Это дополнительно обусловлено необходимостью повысить коэффициент извлечения нефти, а сделать это можно путём максимально детального учёта распределения фильтрационных параметров и получения достоверного прогноза в условиях необходимости наращивания ресурсной базы.

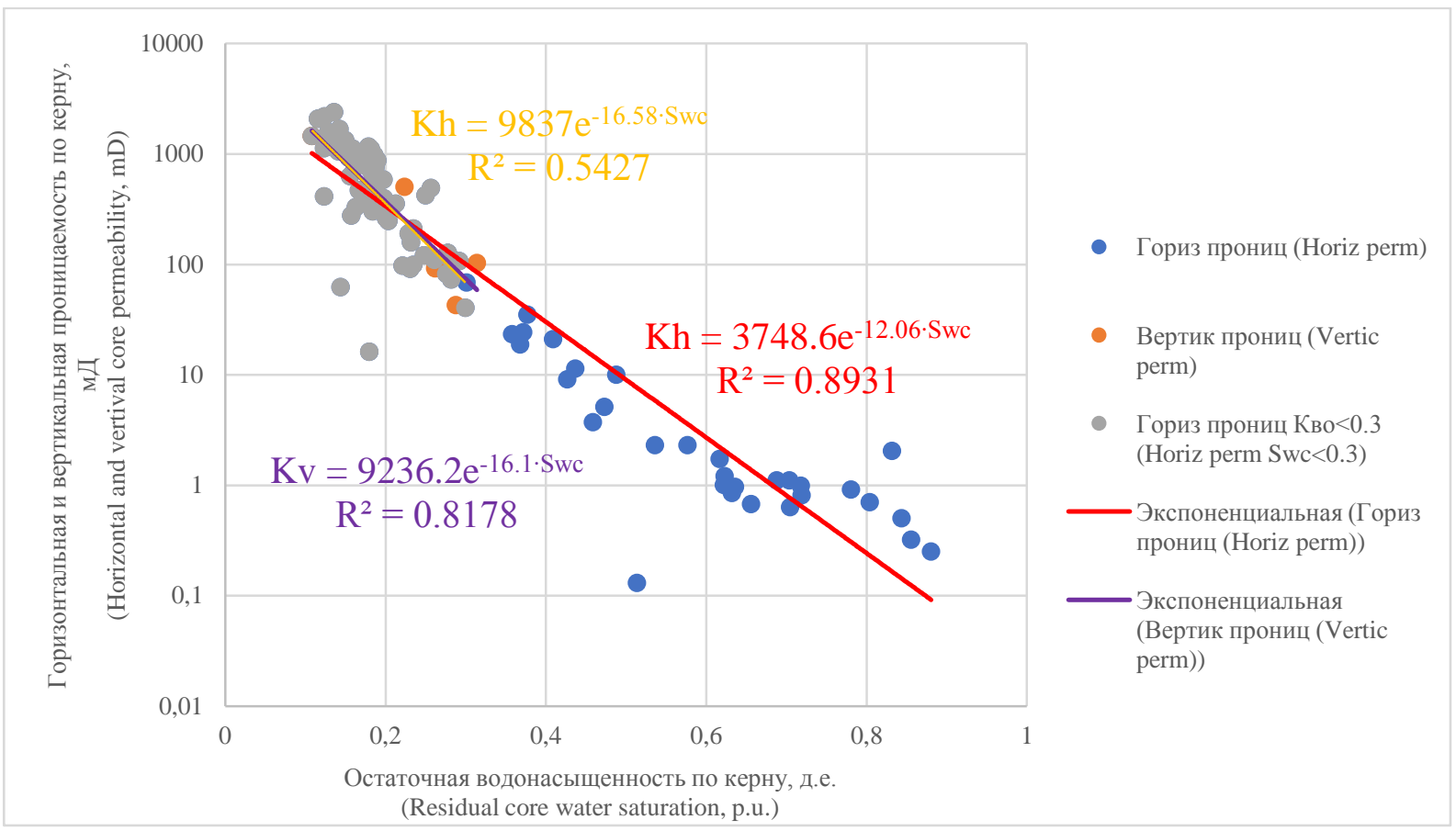

Рис. 3. Зависимость вертикальной и горизонтальной проницаемости от остаточной водонасыщенности по керну

Fig. 3. Vertical and horizontal permeability residual water saturation relationship

Теперь становится возможным распределить проницаемость по вертикали разреза. На полученном распределении видно, что профиль значений вертикальной проницаемости находится чуть ниже профиля значений горизонтальной проницаемости (рис. 4). В некоторых случаях, при моделировании потока флюидов и распределении проницаемости, применяется следующая формула

$$
k_{v}=0,1 \cdot k_{h},
$$

где $k_{v}$ - вертикальная проницаемость, мД; $k_{h}-$ горизонтальная проницаемость, мД.

Из этой формулы становится понятным, что значения вертикальной проницаемости составляют $10 \%$ от значений горизонтальной составляющей. Теперь с помощью фактических данных по керну появилась 
возможность выявить формулу, более приближенную к реальности (рис. 2). Соответственно, в дальнейшем это приведёт к снижению неопределённостей в прогнозе проницаемости в пространстве коллектора.

Таким образом, предыдущие параметры анизотропной модели остаются прежними, а именно, направление фильтрации флюидов и соотношение максимального и минимального значений горизонтальной проницаемости. В данной работе добавляется вертикальная проницаемость и проводятся расчёты.

Непосредственно на данном месторождении учтено влияние анизотропии порового пространства и проницаемости по керновым исследованиям, геофизическим исследованиям скважин, индикаторным (трассерным) исследованиям.
Фактически на данный момент существует методика учёта анизотропии проницаемости в горизонтальной плоскости [23]. Но структура коллектора более сложная и многогранная, чем она описывается текущими методиками и подходами. Одной из целей подобной работы и исследований является максимально объективно приблизиться к увеличению нефтедобычи (коэффициента извлечения нефти) на стадии проектирования разработки именно в том временном промежутке, когда есть возможность учесть все возможные факторы, влияющие да поток флюидов. Дополнительным благоприятным фактором является то, что современное программное обеспечение позволяет сделать детальнейшую модель с учётом даже малейших изменений коллекторского пространства.

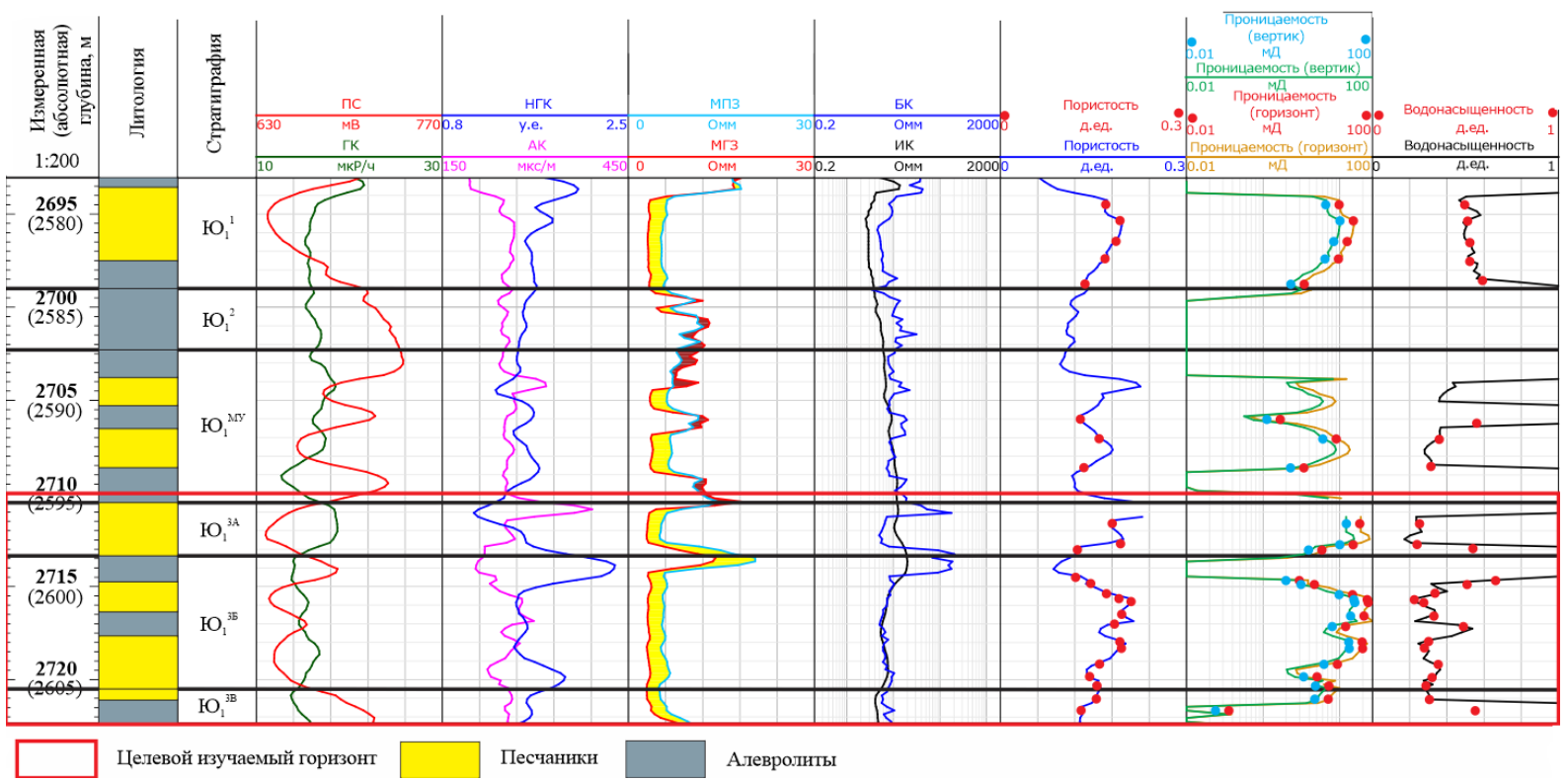

Pис. 4. Планшет интерпретаџии типовой скважины исследуемого месторождения

Fig. 4. Typical well log plot

Наличие данных для дополнительных расчётов, их полнота и качество позволяют взять определённый объект для исследований. Выбор объекта был произведён на основании работ [23-26]. Дополнительные причины - наличие кернового материала для расчётов и тот факт, что по этому месторождению уже проведены расчёты по учёту горизонтальной анизотропии проницаемости. Таким образом, происходит дополнение и приближение к реальности распределения петрофизических свойств.

Изучаемое месторождение является сложнопостроенным, осложнённым тектоническими процессами. Дополнительно своё влияние оказывают седиментационные процессы, происходившие в момент осадконакопления, дальнейшего погружения осадка и формирования нефтяной залежи. Для полноты и достоверности исследований были проведены расчёты по 16 моделям (по полигонам 8, по участкам 6, по месторождению 2) [26]. Ретроспективные данные разработки можно проследить по графику на рис. 5. На нём отражено сравнение расчётных параметров накопленной добычи нефти и фактических по всему месторождению.
Полученные результаты показывают, что фактические данные по добыче и расчёты по гидродинамическим моделям не только сопоставимы, но и характеризуются малыми отклонениями значений друг от друга. Погрешность расчётов составляет 3,09 \%, что на $0,69 \%$ ниже погрешности при учёте только горизонтальной анизотропии проницаемости. Таблица наглядно отражает ошибки сопоставления расчётных данных и данных добычи.

Таблица. Расхождения в данных фактической добычи и расчётных параметрах

Table. Calculations and actual data comparison

\begin{tabular}{|c|c|c|}
\hline \multicolumn{3}{|c|}{ Ошибка, \%/Error, \% } \\
\hline & Анизотропная & Анизотропная модель \\
Изо- & модель (только & (горизонтальная \\
тропная & горизонтальная & и вертикальная \\
модель & проницаемость) & проницаемость) \\
Isotropic & Anisotropic model & Anisotropic model \\
model & (only horizontal & (horizontal and vertical \\
& permeability) & permeability) \\
\hline 9,41 & 3,78 & 3,09 \\
\hline
\end{tabular}




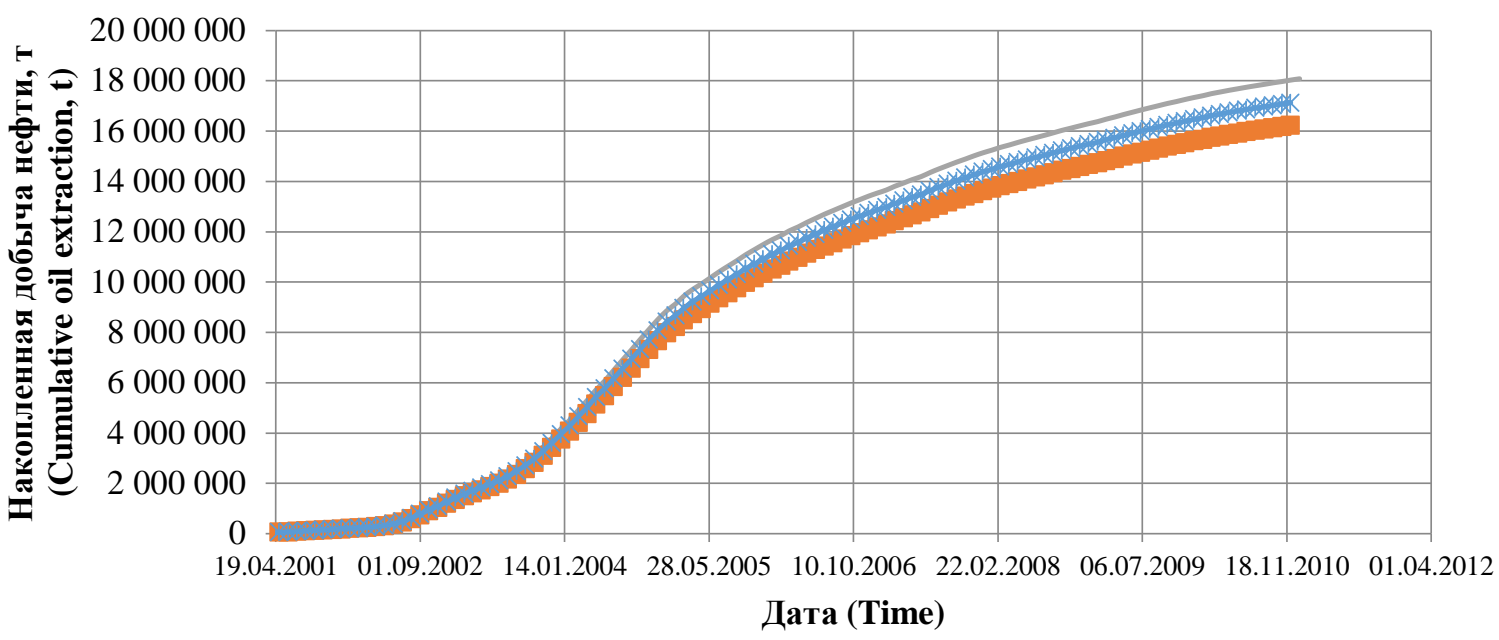

- Накопленная добыча нефти, т - - Изотропная $\quad$ * Анизотропная

Pис. 5. Сопоставление фактически накопленной добычи нефти с накопленной добычей по изотропной и анизотропной моделям, учитывающим горизонтальную и вертикальную пронииаемости,

Fig. 5. Cumulative oil extraction (isotropic, anisotropic models including horizontal and vertical permeability)

Проведённый анализ показывает, что даже при практически равных значениях вертикальной и горизонтальной проницаемости анизотропная модель более приближена к фактическим результатам разработки, чем изотропная, а в случае различия проницаемостей эффективность прогноза анизотропной модели может быть более существенной.

\section{Заключение и выводы}

Полученные результаты свидетельствуют о необходимости обязательного учёта вертикальной проницаемости при моделировании нефтяных месторождений. В особенности, когда это касается создания гидродинамической модели. Учёт влияния компоненты проницаемости в вертикальной плоскости необходим для распределения достоверных значений проницаемости по разрезу и получения гидродинамической модели, на основании которой будут проводиться корректные расчёты добычи углеводородов.

Стандартный набор зависимостей для вертикальной компоненты проницаемости можно получить достаточно быстро. При этом преимущества адаптации

\section{СПИСОК ЛИТЕРАТУРЫ}

1. Interpreting reservoir permeabilities from wireline formation test data / H. Du, Y. Liu, X. Li, F. Guan, X. Sui // Petroleum Science and Technology. - 2013. - V. 31:18. - P. 1819-1826. DOI: 10.1080/10916466.2011.603009

2. Акрам X., Ашуров В. Обзор гидродинамических исследований скважин в открытом и обсаженном стволе модульным испытателем пластов на кабеле MDT/CHDT. - М.: Нефтегазовое обозрение, 2005. - 16 c.

3. Dussan E.B.V., Yogeshwar Sharma. Analysis of the pressure response of a single-probe formation tester // Society of Petroleum Engineers. Formation Evaluation. - Texas, USA, 1 June, 1992. P. 151-156.

4. Permeability anisotropy and its relations with porous medium structure / J.B. Clavaud, A. Maineult, M. Zamora, P. Rasolofosaon, C. Schlitter // Journal of geophysical research. - 2008. - V. 113. P. 1-10.

5. Панков М.В. Анализ разработки Крапивинского нефтяного месторождения // Центр профессиональной переподготовки разработки заключаются в наличии закона (формулы) распределения дополнительного параметра проницаемости, уточнении геологической и гидродинамической моделей, более точном прогнозе характеристик добычи и более эффективном планировании геологотехнических мероприятий и применения методов увеличения нефтеотдачи.

Остаётся проблема недостаточности или отсутствия данных для построения соответствующих зависимостей вертикальной проницаемости от пористости или горизонтальной проницаемости. Для решения подобной проблемы можно для близлежащих месторождений использовать изучаемое месторождение в качестве опорного, и для месторождений с аналогичными условиями осадконакопления - в качестве месторождения-аналога. Также необходимо провести комплексный анализ распределения значений вертикальной проницаемости на разных месторождениях. Это позволит посмотреть на распределение параметров со стороны изменения фациальной обстановки осадконакопления и седиментационных процессов.

специалистов нефтегазового дела: отчёт. - Томск: Изд-во НИ ТПУ, 2004. -425 c.

6. Меркулов В.П., Краснощекова Л.А. Исследование пространственной литолого-петрофизической неоднородности продуктивных коллекторов месторождений нефти и газа // Известия Томского политехнического университета. - 2002. - Т. 305. № 6. - C. 296-303.

7. Tyvand P.A., Storhaug A.R.F. Two-dimensional flow in a porous medium with general anisotropy. - Trondheim: Department of Mathematical Sciences and Technology (Norwegian University of Life Sciences), 2004. $-20 \mathrm{p}$.

8. Литолого-петрофизическая анизотропия песчано-глинистых коллекторов нефтяных месторождений / В.П. Меркулов, Д.В. Александров, Л.А. Краснощекова, Ю.Я. Ненахов // Научно-технический вестник ЮКОС - 2004 - № 10. - С. 33-36.

9. Abedi R., Clarke P.L. Modeling of rock inhomogeneity and anisotropy by explicit and implicit representation of microcracks // 52nd U.S. Rock Mechanics/Geomechanics Symposium. - Seattle, Washington, USA, 17-20 June, 2018. - P. 1-11. 
10. Zhang Z., Du J. Reservoir characterization using perforation shots: anisotropy and attenuation // SEG International Exposition and Annual Meeting. - Anaheim, California, USA, 14-19 October, 2018. - P. 2982-2986.

11. Kolbikov S., Kuznetsova Y., Smirnov A. Method of anisotropy modeling and its application to hydrodynamic simulation // SPE Russian Petroleum Technology Conference. - Moscow, Russia, 15-17 October, 2018. - P. 1-9.

12. Makhenko R.Y., Tarokh A. Anisotropy in the undrained pore pressure response of rock // 52nd U.S. Rock Mechanics/Geomechanics Symposium. - Seattle, Washington, USA, 17-20 June, 2018. - P. 40-51.

13. Liu S., King M.J. Improved calculation of effective permeability for pore network models using the diffuse source methodology // 81st EAGE Conference and Exhibition. - London, England, UK, 3-6 June, 2019. - P. 1-19.

14. Gray D.H., Fatt I., Bergamini G. The effect of stress on permeability of sandstone cores // Society of petroleum engineers' journal. - 1963. - V. 3. - P. 95-99.

15. Wannell M.J., Colley N.M., Halford F.R. The use of a new technique to determine permeability anisotropy // Society of Petroleum Engineers. - Schlumberger Evaluation \& Production Services. - Aberdeen, United Kingdom, 7 September, 1993. V. 9. - P. 489-495

16. Cosan A. Measuring permeability anisotropy: the latest approach // Oilfield Review. - 1994. - V. 6. - № 4. - P. 24-35.

17. Carpenter C. Better permeability estimation from wireline formation testing // Journal of Petroleum Technology. - 2018 February. - V. 70. - P. 66-68

18. Pan Y., Medhat M.K., Wayne N. Fieldwide determination of directional permeabilities using transient well testing // SPE Reservoir Evaluation \& Engineering. - 2019 May. - V. 22. - P. 1-11

19. Жабрев И.П., Стуканогов Ю.А. Зависимость нефтеотдачи от направления вытеснения нефти водой в анизотропных по проницаемости пластах // Геология нефти и газа. - 1992. № 8. - C. 34-36.

20. Al-Hadrami H.K., Teufel L.W. Influence of permeability anisotropy and reservoir heterogeneity on optimization of infill drilling in naturally fractured tight-gas Mesaverde sandstone reservoirs, San Juan Basin // SPE Low Permeability Reservoirs Symposium. - Denver, U.S.A., March 12-15, 2000. - P. 1-12.

21. Батлер Р.М. Горизонтальные скважины для добычи нефти, газа и битумов. - М.; Ижевск: Институт компьютерных исследований, НИЦ «Регулярная и хаотическая динамика», 2010. $536 \mathrm{c}$

22. Geological factors influencing reservoir performance of the Hartzog Draw field / C.L. Hearn, W.J. Ebanks Jr., R.S. Tye, V. Ranganathan. - San Francisco: SPE annual technical conference, 1983. - P. 1335-1344.

23. Коровин М.О. Специализированный анализ керна для изучения анизотропии коллекторов нефти и газа // Геофизика. 2014. - Т. 324. - № 1. - C. 87-92.

24. Конторович В.А. Тектоника и нефтегазоносность мезозойскокайнозойских отложений юго-восточных районов Западной Сибири. - Новосибирск: СО РАН «Гео», 2002. - 253 с.

25. Роль мезозойско-кайнозойской тектоники в формировании залежей углеводородов в южных частях Каймысовского свода и Нюрольской мегавпадины / В.А. Конторович, М.В. Соловьев, Л.М. Калинина, А.Ю. Калинин // Геология и геофизика. 2011. - T. 8. - C. 1075-1091.

26. Коровин М.О. Методика количественной оценки латеральной анизотропии фильтрационно-ёмкостных свойств терригенных коллекторов на базе комплексного анализа геологогеофизических данных: дис. ... канд. геол.-минерал. наук. Томск, 2017. - $108 \mathrm{c}$.

Поступила 23.12.2020 2.

\section{Информация об авторах}

Коровин M.O., кандидат геолого-минералогических наук, доцент отделения нефтегазового дела Инженерной школы природных ресурсов Национального исследовательского Томского политехнического университета. 
UDC550.3

\title{
VERTICAL PERMEABILITY EFFECT ON TERRIGENEOUS OIL FIELD RESERVOIR ADAPTATION
}

\author{
Mikhail O. Korovin, \\ korovinmo@hw.tpu.ru \\ National Research Tomsk Polytechnic University, \\ 30, Lenin avenue, Tomsk, 634050, Russia.
}

The relevance of the research lies in the need to create an algorithm for calculating permeability in three-dimensional space using geological and geophysical data. At this stage, the horizontal distribution of permeability should be taken into account. And the vertical permeability is usually set by the ratio of the permeability in the horizontal plane and the decreasing or increasing coefficient. In fact, in this case, the spatial change in the vertical component of the permeability along the section is not taken into account. This happens for two reasons: a small number of studies of vertical permeability and, accordingly, the absence of equations for calculating this very important parameter. This is a global problem of lack of initial information when examining actual core material. And the core material is the basis for studying the anisotropic characteristics of rocks. Permeability, in its turn, is the most important reservoir characteristic and affects the ability to extract hydrocarbons from reservoir strata.

The aim of this work is to form a dependence of vertical permeability on porosity, the distribution of permeability in the geological and hydrodynamic model, check the quality of adaptation of production and injection data.

The study object is a field with a terrigenous reservoir type. In this field, calculations of the anisotropy of horizontal permeability have already been carried out, and within the framework of this study, calculations of the vertical permeability were carried out and its influence on the adaptation of the field was taken into account. A feature of this field is the variability of the direction of the improved permeability zones, as well as the presence of laboratory core studies of the vertical permeability of reservoirs. Such studies will allow determining the vertical permeability of rocks and calculating the vertical distribution of the section. The studied formations are represented by Upper Jurassic deposits with a non-uniform distribution of filtration and reservoir properties.

Methods: plotting the dependence of vertical permeability on porosity based on core studies, introducing the vertical component of permeability into geological and hydrodynamic models, calculating the adaptation of actual production data after taking into account the vertical component of permeability.

As a result of the study, the petrophysical model of the studied field was refined, it turned out to achieve a more accurate distribution of petrophysical properties in the geological model. Thus, it becomes possible to take into account the real features of the geological structure, hence all three components of the permeability vector are used. One of the main points is the creation of a model that describes the distribution of properties as accurately as possible and reflects the real characteristics of production. Carrying out the most accurate forecast and planning the application of enhanced oil recovery methods. A huge amount of accumulated data makes it possible to create correlations between various petrophysical parameters. It is necessary to supplement and develop a database, which in the near future can serve as a storage for analogous fields that do not have the data to create their own similar dependencies.

\section{Key words:}

Permeability anisotropy, anisotropy scale, vertical anisotropy, hydrodynamic simulation, petrophysical calculations.

\section{REFERENCES}

1. Du H., Liu Y., Li X., Guan F., Sui X. Interpreting reservoir permeabilities from wireline formation test data. Petroleum Science and Technology, 2013, vol. 31:18, pp. 1819-1826. DOI: 10.1080/10916466.2011.603009

2. Akram H., Ashurov V. Obzor gidrodinamicheskih issledovaniy skvazhin $v$ otkrytom $i$ obsazhennom stvole modul'nym ispytatelem plastov na kabele MDT/CHDT [Review of well testing in open and cased hole with MDT / CHDT modular reservoir tester]. Moscow, Neftegazovoe obozrenie Publ., 2005. 16 p.

3. Dussan E.B.V., Yogeshwar Sharma Analysis of the Pressure Response of a Single-Probe Formation Tester. Society of Petroleum Engineers. Formation Evaluation. Texas, USA, 1 June, 1992. pp. $151-156$.

4. Clavaud J.B., Maineult A., Zamora M. Rasolofosaon P., Schlitter C. Permeability anisotropy and its relations with porous medium structure. Journal of geophysical research, 2008, vol. 113, pp. 1-10.

5. Pankov M.V. Analiz razrabotki Krapivinskogo neftyanogo mestorozhdeniya [Analysis of the development of the Krapivinskoye oil field]. Centr professionalnoy perepodgotovki spetsialistov neftegazovogo dela: otchet [Center for training and retraining of oil and gas specialists: report]. Tomsk, TPU Publ. house, 2004, $425 \mathrm{p}$.

6. Merkulov V.P., Krasnoshchekova L.A. Study of spatial lithological and petrophysical heterogeneity of productive reservoirs of oil and gas fields. Bulletin of the Tomsk Polytechnic University, 2002, vol. 305, no. 6, pp. 296-303. In Rus.
7. Tyvand P.A., Storhaug A.R.F. Two-dimensional flow in a porous medium with general anisotropy. Trondheim, Department of Mathematical Sciences and Technology (Norwegian University of Life Sciences), 2004. 20 p.

8. Merkulov V.P., Aleksandrov D.V., Krasnoshchekova L.A., Nenahov Y.U. Lithological-petrophysical anisotropy of sandyargillaceous reservoirs of oil fields. Nauchno-tekhnicheskiy vestnik YUKOS, 2004, no. 10, pp. 33-36. In Rus.

9. Abedi R., Clarke P.L. Modeling of rock inhomogeneity and anisotropy by explicit and implicit representation of microcracks. $52^{\text {nd }}$ U.S. Rock Mechanics/Geomechanics Symposium. Seattle, Washington, USA, 17-20 June, 2018. pp. 1-11.

10. Zhang Z., Du J. Reservoir characterization using perforation shots: anisotropy and attenuation. SEG International Exposition and Annual Meeting. Anaheim, California, USA, 14-19 October, 2018. pp. 2982-2986.

11. Kolbikov S., Kuznetsova Y., Smirnov A. Method of Anisotropy Modeling and its Application to Hydrodynamic Simulation. SPE Russian Petroleum Technology Conference. Moscow, Russia, 15-17 October, 2018, pp. 1-9.

12. Makhenko R.Y., Tarokh A. Anisotropy in the undrained pore pressure response of rock. $52^{\text {nd }}$ U.S. Rock Mechanics/Geomechanics Symposium. Seattle, Washington, USA, 17-20 June, 2018. pp. 40-51.

13. Liu S., King M.J. Improved calculation of effective permeability for pore network models using the diffuse source methodology. $81^{s t}$ EAGE Conference and Exhibition. London, England, UK, 3-6 June, 2019. pp. 1-19. 
14. Gray D.H., Fatt I., Bergamini G. The effect of stress on permeability of sandstone cores. Society of petroleum engineers' journal, 1963, vol. 3, pp. 95-99.

15. Wannell M.J., Colley N.M., Halford F.R. The use of a new technique to determine permeability anisotropy. Society of Petroleum Engineers. Schlumberger Evaluation \& Production Services. Aberdeen, United Kingdom, 7 September, 1993. Vol. 9, pp. 489-495.

16. Cosan A. Measuring permeability anisotropy: the latest approach. Oilfield Review, 1994, vol. 6, no. 4, pp. 24-35.

17. Carpenter C. Better permeability estimation from wireline formation testing. Journal of Petroleum Technology. February, 2018, vol. 70, pp. 66-68.

18. Pan Y., Medhat M.K., Wayne N. Fieldwide determination of directional permeabilities using transient well testing. SPE Reservoir Evaluation \& Engineering, May, 2019, vol. 22, pp. 1-11.

19. Zhabrev I.P., Stukanogov Yu.A. Zavisimost nefteotdachi ot napravleniya vytesneniya nefti vodoy $\mathrm{v}$ anizotropnykh po pronitsaemosti plastakh [Dependence of oil recovery on the direction of oil displacement by water from reservoirs anisotropic in permeability]. Geologiya nefti i gaza, 1992, no. 8, pp. 34-36.

20. Al-Hadrami H.K., Teufel L.W. Influence of permeability anisotropy and reservoir heterogeneity on optimization of infill drilling in naturally fractured tight-gas Mesaverde sandstone reservoirs, San Juan Basin. SPE Low Permeability Reservoirs Symposium. Denver, U.S.A., March 12-15, 2000. pp. 1-12.

21. Batler R.M. Gorizontalnye skvazhiny dlya dobychi nefti, gaza i bitumov [Horizontal wells for oil, gas and bitumen production].
Moscow, Izhevsk, Computer Research Institute Publ., NIC «Regulyarnaya i khaoticheskaya dinamika», $2010.536 \mathrm{p}$.

22. Hearn C.L. Ebanks W.J. Jr., Tye R.S., Ranganathan V. Geological factors influencing reservoir performance of the Hartzog Draw field. SPE annual technical conference. San Francisco, 1983. pp. $1335-1344$.

23. Korovin M.O. Specialized analysis of core for study of anisotropy of oil and gas collectors. Geophysics, 2014, vol. 324, no. 1, pp. 87-92. In Rus.

24. Kontorovich V.A. Tektonika $i$ neftegazonosnost mezozoyskokaynozoyskikh otlozheniy yugo-vostochnykh rayonov Zapadnoy Sibiri [Tectonics and oil and gas content of the MesozoicCenozoic deposits of the southeastern regions of Western Siberia]. Novosibirsk, SO RAN «Geo» Publ., 2002. 253 p.

25. Kontorovich V.A. Solovev M.V., Kalinina L.M., Kalinin A.Yu. The role of the Mesozoic-Cenozoic tectonics in the formation of hydrocarbon deposits in the southern parts of the Kaimysov arch and the Nyurol megadepression. Geology and Geophysics, 2011, vol. 8, pp. 1075-1091. In Rus.

26. Korovin M.O. Metodika kolichestvennoy otsenki lateralnoy anizotropii filtratsionno-emkostnykh svoystv terrigennykh kollektorov na baze kompleksnogo analiza geologo-geofizicheskikh dannykh. Diss. Kand. nauk [Methodology for quantitative assessment of lateral anisotropy of filtration-capacity properties of terrigenous reservoirs based on a comprehensive analysis of geological and geophysical data. Cand. Diss.]. Tomsk, 2017. 108 p.

Received: 23 December 2020.

\section{Information about the authors}

Mikhail O. Korovin, Cand. Sc., associate professor, National Research Tomsk Polytechnic University. 